Supplementary information

\title{
Substrate Lattice Guided $\mathrm{MoS}_{2}$ Crystal Growth:
}

\author{
Implications for van der Waals epitaxy \\ Yung-Yu Lai ${ }^{1}$, Chi-Huang Chuang ${ }^{2}$, Yen-Wei Yeh ${ }^{3}$, Cheng-Hung Hou ${ }^{2}$, Shih-Chieh \\ $\mathrm{Hsu}^{4}$, Yi Chou ${ }^{5}$, Yi-Chia $\mathrm{Chou}^{5}$, Hao-Chung $\mathrm{Kuo}^{3}$, YewChung Sermon $\mathrm{Wu}^{1}$, and \\ Yuh-Jen Cheng*2,3 \\ ${ }^{1}$ Department of Materials Science and Engineering, National Chiao Tung University, Hsinchu 300, \\ Taiwan \\ ${ }^{2}$ Research Center for Applied Sciences, Academia Sinica, 128 Sec. 2, Academia Rd, Nankang, Taipei \\ 115, Taiwan \\ ${ }^{3}$ Department of Photonics and Institute of Electro-Optical Engineering, National Chiao Tung University, Hsinchu 300, Taiwan \\ ${ }^{4}$ Department of Chemical and Materials Engineering, Tamkang University, New Taipei City 25137, \\ Taiwan \\ ${ }^{5}$ Department of Electrophysics, National Chiao Tung University, Hsinchu 300, Taiwan
}

yjcheng@sinica.edu.tw

\section{S1 2" Sapphire substrate orientation.}

The 2" sapphire substrate is a c-plane epi-ready substrate with an orientation flat in the a-plane [11 $\overline{2} 0]$ direction. The substrate was diced into $1 \mathrm{~cm} \mathrm{x} \sim 5 \mathrm{~cm}$ rectangles (dotted rectangle in Figure S1) for $\mathrm{MoS}_{2}$ growth. Given the orientation flat reference, we can identify the grown $\mathrm{MoS}_{2}$ crystal orientation with respect to sapphire substrate crystal orientation. 


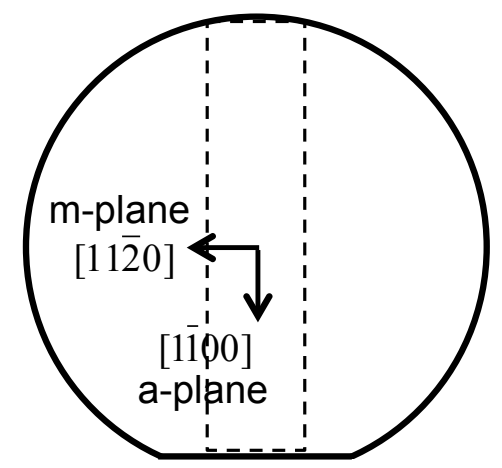

Figure S1. Crystal orientation of c-plane sapphire substrate used in the experiment.

\section{S2 Optical images of $\mathrm{MoS}_{2}$ film and XPS analysis}

Figure S2a shows the optical microscope images of a continuous $\mathrm{MoS}_{2}$ film on a sapphire substrate at locations I-VI, as depicted in Figure 1a inset. They show almost featureless smooth surface except at substrate location VI. The light color patches in VI are thick MoS-Ox due to sulfurized thick MoOx or multiple layer MoS2-Ox, which is also visible at the center of a $\mathrm{MoS}_{2}$ crystal shown in Figure 1c(VI).
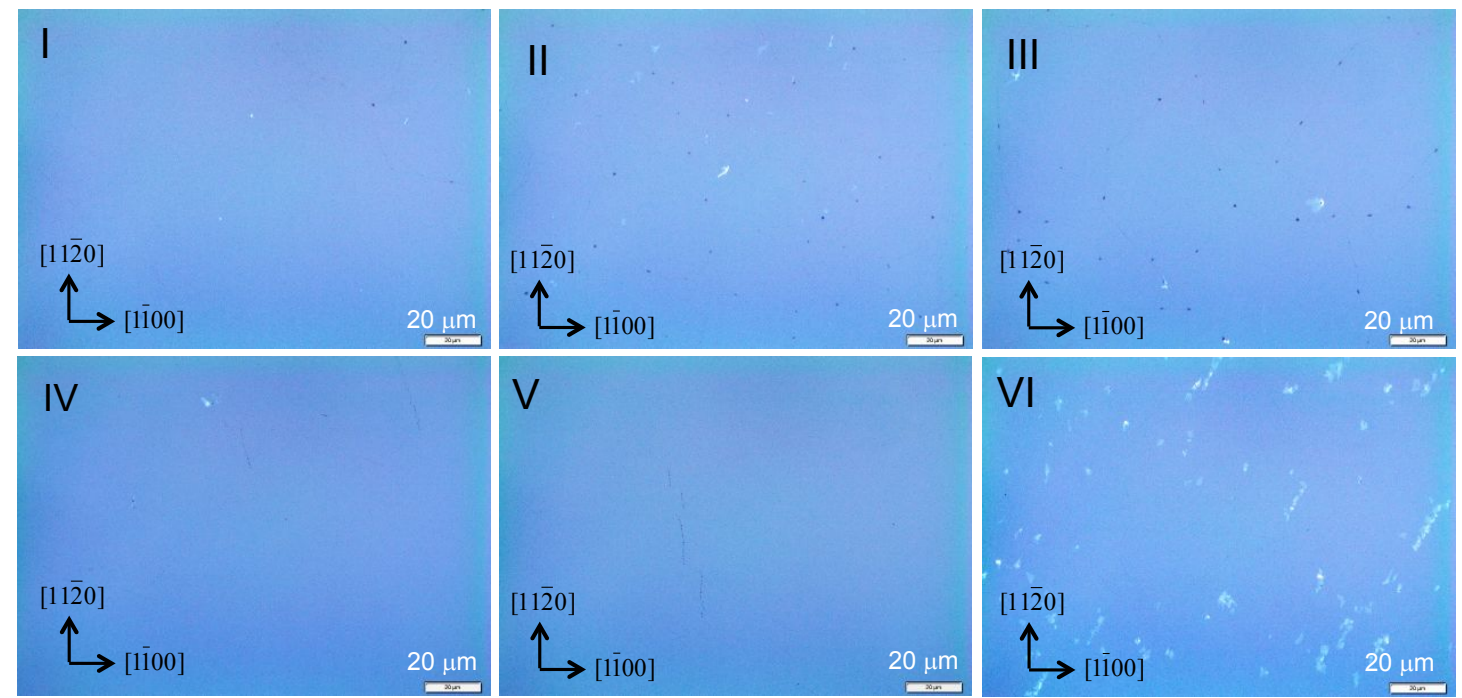

Figure S2a. Optical images of MoS2 film at substrate locations I-VI, as depicted in Figure 1a inset.

XPS analysis of $\mathrm{MoS}_{2}$ crystal in Figure 1c shows some residual $\mathrm{Mo}^{6+}$ signal. Atomic concentration \% analysis shows Mo : $\mathrm{S}=1.03: 2$ ratio. Mo concentration (33.92\%) is slightly larger than half of the $\mathrm{S}$ concentration $(66.08 \% / 2=33.04 \%)$. The excess amount is due to the presence of $\mathrm{Mo}^{6+}$ signal, indicating there are some residual MoOx on substrate. 

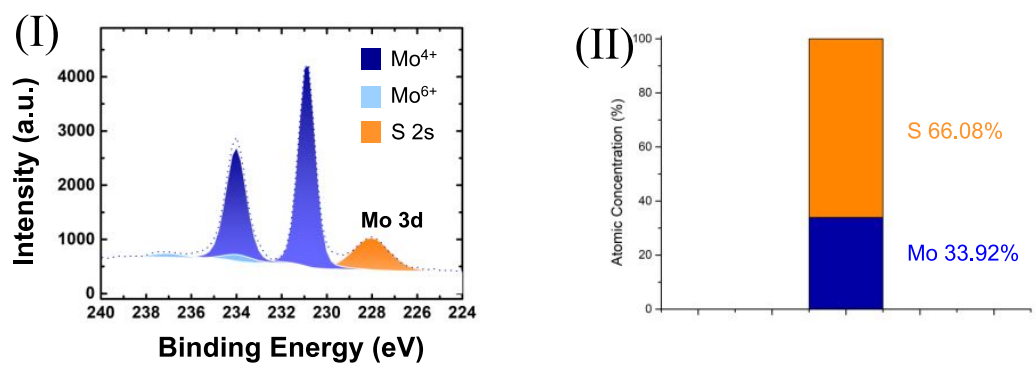

Figure S2b. (I) XPS analysis of grown $\mathrm{MoS}_{2}$ crystal. (II) Surface element concentration percentage from XPS analysis.

\section{S3 XPS analysis of Mo foil}

The surface element of Mo foil was analyzed by XPS after three different growth processes with different oxygen flow. The purpose is to investigate the surface element after $500{ }^{\circ} \mathrm{C}$ oxidation period, as shown in Figure S3a, after a complete growth run with continuous $2 \mathrm{sccm}$ oxygen flow, as shown in Figure S3b, and after a complete growth run with 2 sccm oxygen flow turned off during growth period at 790 ${ }^{\circ} \mathrm{C}$, as shown in Figure S3c. The surface element composition concentration for these different processes is shown in Figure S5.

(a)

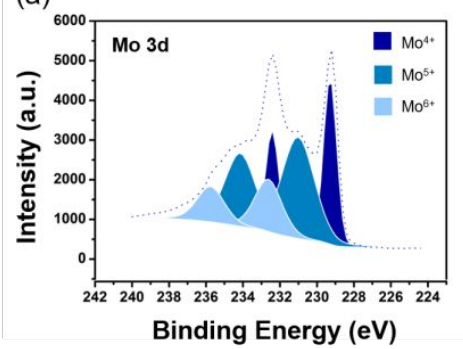

(b)

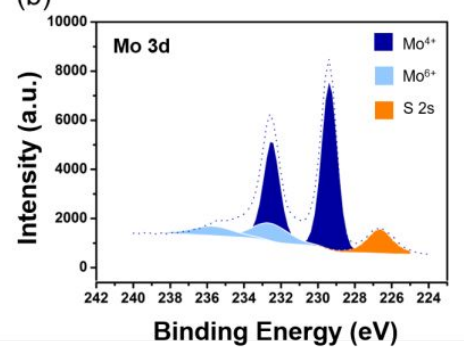

(c)

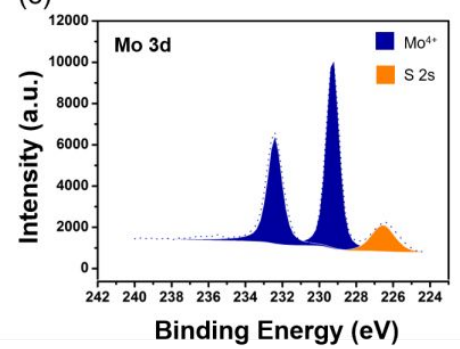

Figure S3. Mo 3d XPS spectrum acquired from Mo foil at different stage of CVD growth. (a) Growth process terminated at the end of $500{ }^{\circ} \mathrm{C}$ oxidation with $2 \mathrm{sccm}$ oxygen flow. (b) Growth process with continuous $2 \mathrm{sccm}$ oxygen flow. (c) Growth process with 2 sccm oxygen flow turned off during growth period at temperature 790 ${ }^{\circ} \mathrm{C}$. 

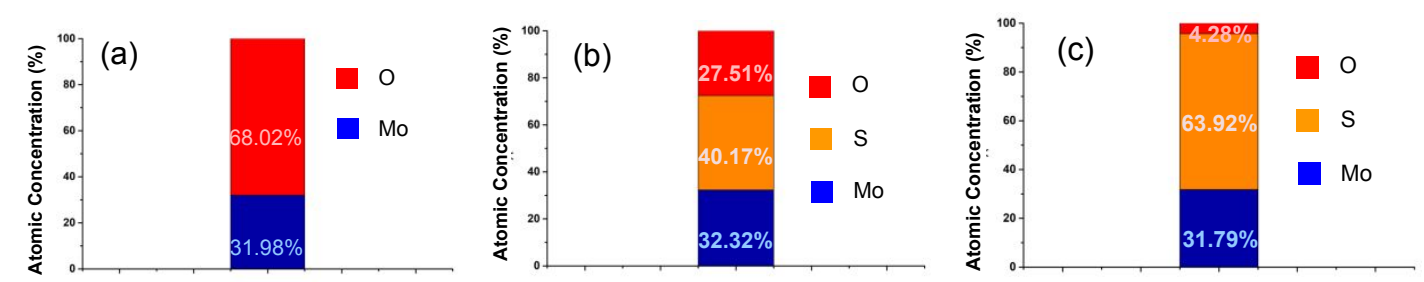

Figure S4. Surface element concentration percentage from the XPS analysis of Mo foil (a) Growth process terminated at the end of $500{ }^{\circ} \mathrm{C}$ oxidation with $2 \mathrm{sccm}$ oxygen flow. (b) Growth process with continuous 2 sccm oxygen flow. (c) Growth process with $2 \mathrm{sccm}$ oxygen flow turned off during growth period at temperature $790{ }^{\circ} \mathrm{C}$.

According to Figure S3a and S4a, the Mo foil was mostly covered by Mo oxide after the $500{ }^{\circ} \mathrm{C}$ oxidation period. Several oxidation states of $\mathrm{Mo}$, including $\mathrm{Mo}^{4+}, \mathrm{Mo}^{5+}$ and $\mathrm{Mo}^{6+}$, were identified but none of these oxides contributed to $\mathrm{MoS}_{2}$ crystal formation because there is no sulfur element signal. When $2 \mathrm{sccm}$ oxygen flow continues during the whole growth process, Figure S3b and S4b show that Mo foil surface was covered by Mo oxide and $\mathrm{MoS}_{2}$. The significant amount of Mo oxide indicates that Mo foil could still serve as a $\mathrm{MoOx}$ source for $\mathrm{MoS}_{2}$ crystal growth under continuous oxygen flow. When the 2 sccm oxygen flow was turned off during growth period at $790{ }^{\circ} \mathrm{C}$, Figure S3c and S4c show that Mo foil was almost fully covered by $\mathrm{MoS}_{2}$ after growth process. Mo foil surface was capped by $\mathrm{MoS}_{2}$, which indicates that the Mo oxide evaporation stopped early. As a result, the MoS2 crystal growth is terminated early due to the shortage of MoOx supply.

\section{S4 Crystal growth aligned to $[1 \overline{100}]$ direction}

Following Figure S5a-S5d are the SEM images of grown $\mathrm{MoS}_{2}$ crystals when $2 \mathrm{sccm}$ oxygen flow continues through out the whole growth process. The scale bars vary from 50 to $1 \mu \mathrm{m}$. The densely populated $\mathrm{MoS}_{2}$ crystals are all aligned with triangle

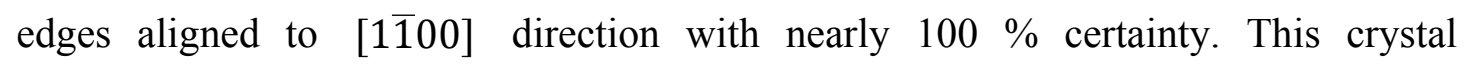
morphology extends to whole sapphire substrate surface except at the edges. 

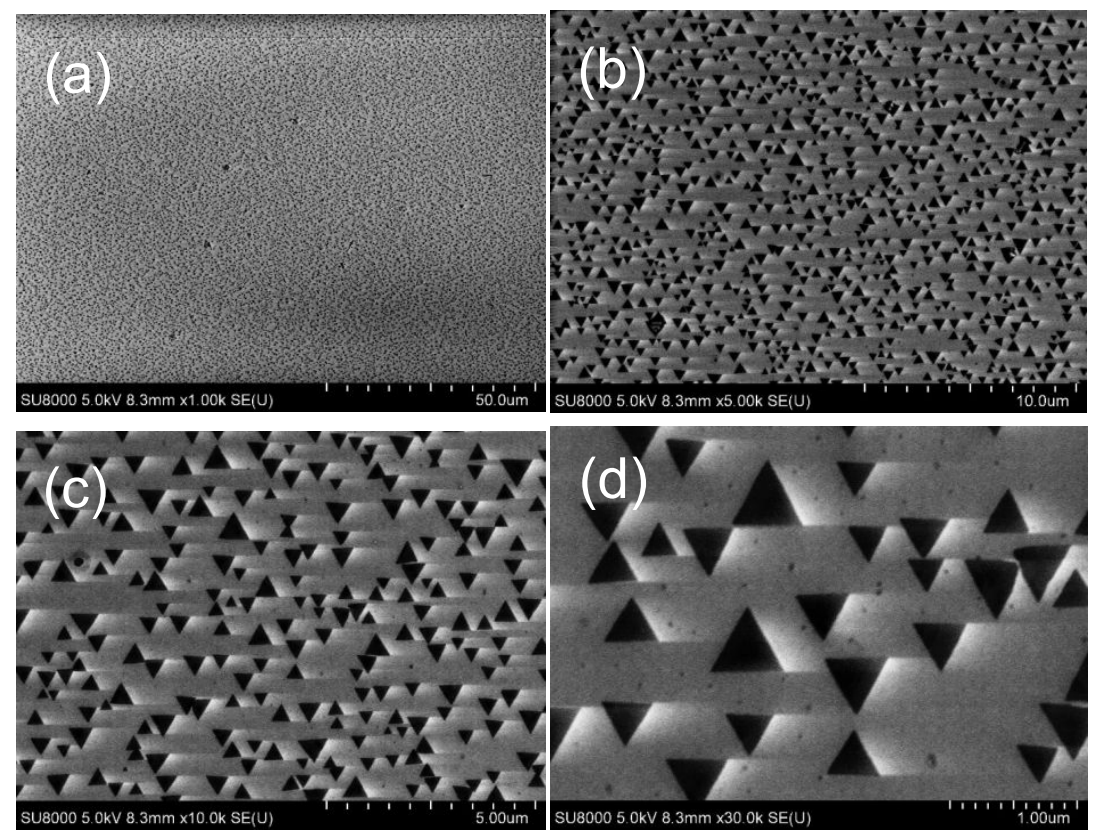

Figure S5. (a)-(d) SEM images of aligned $\mathrm{MoS}_{2}$ crystal growth at different magnification showing the near perfect growth alignment, where $2 \mathrm{sccm}$ oxygen flow continues through out the whole growth process. This alignment covers nearly the full $5 \mathrm{~cm}$ length of sapphire substrate.

\section{S5 XPS analysis of $\mathrm{MoS}_{2}$ crystals in Figure 3}

XPS analysis of $\mathrm{MoS}_{2}$ crystals in Figure 3 shows some $\mathrm{Mo}^{6+}$ signal. From atomic concentration \% analysis, it indicates $\mathrm{Mo}: \mathrm{S}=1.18: 2$ ratio. Mo concentration $(37.23 \%)$ is larger than half of the $\mathrm{S}$ concentration $(62.77 \% / 2=31.38 \%)$. The excess Mo amount is attributed to some $\mathrm{MoOx}$ on substrate due to excess $\mathrm{MoOx}$ supply.
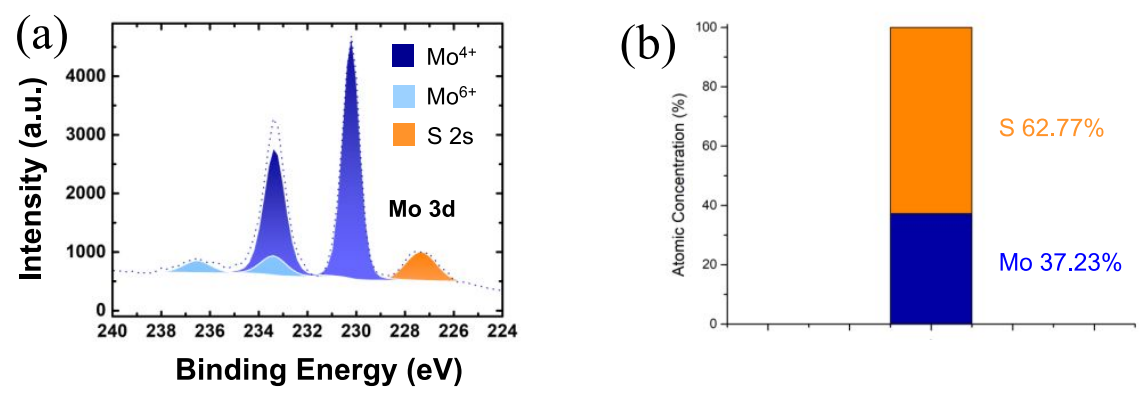

Figure S6. (a) XPS analysis of $\mathrm{MoS}_{2}$ in Figure 3. (b) Surface element concentration percentage from the XPS analysis. 